\title{
Assessment of Hepatic Function, Operative Candidacy, and Medical Management after Liver Resection in the Patient with Underlying Liver Disease
}

\author{
Gebhard Wagener, MD ${ }^{1}$ \\ ${ }^{1}$ Department of Anesthesiology, College of Physicians and Surgeons, \\ Columbia University, New York \\ Semin Liver Dis 2013;33:204-212. \\ Address for correspondence Gebhard Wagener, MD, Department of \\ Anesthesiology, Columbia University, P and S Box 46 (PH-5) 630 West \\ 168th Street, New York, NY 10032-3784 \\ (e-mail: gw72@columbia.edu).
}

\begin{abstract}
Liver resection in patients with underlying liver disease remains a formidable challenge. It requires adequate patient selection, a precise surgical plan, and avoidance of additional ischemic insults during surgery. Precise estimation of the residual liver volume using computed tomography or magnetic resonance imaging and computerassisted volumetry allows the calculation of residual to total liver volume (RLV/TLV) ratios. Although RLV/TLV ratios over 20 to $25 \%$ are considered sufficient in healthy livers, patients with cirrhosis may only tolerate resections that result in RLV/TLV ratios over $40 \%$ and higher. Conventional laboratory tests may not be able to sufficiently predict liver reserve after resection. Dynamic tests such as indocyanine green clearance have been used to assess residual liver function and assist in deciding about operability of patients with underlying liver disease undergoing extensive resections. Intraoperative management should focus on avoiding blood loss and ischemic injury to the liver. Low central

Keywords

- hepatectomy

- liver function

- cirrhosis

- liver tumors

- dynamic liver tests

- liver injury venous pressure may reduce blood loss and is recommended if tolerated without impeding renal perfusion. Portal vein and hepatic artery occlusion during resection can reduce blood loss, but will cause ischemic insult to the liver that may jeopardize residual liver function and induce postoperative hepatic failure. When feasible, vascular occlusion should be avoided in patients with underlying liver disease. The postoperative recovery is usually fast if sufficient liver remains. However, vigilance is required to detect liver dysfunction and treat its complications.
\end{abstract}

\section{Preoperative Assessment}

Evaluation of the Required Extent of Liver Resection Most hepatic resections are performed as treatment for either primary liver tumors or metastasis secondary to extrahepatic tumors. These resections need to have a sufficient margin in order to be curative; therefore, the precise location of the tumor is necessary prior to surgery. It is further mandatory to estimate the remaining liver size to avoid insufficient functional reserve of the liver remnant after resection. Estimation of the remaining liver function after resection can be complicated by baseline liver disease such as cirrhosis. Hepatic cirrhosis not only decreases the functional reserve of the remaining liver, but also renders is succinctly sensitive to ischemic injury. Preoperative estimation of remaining liver size is the first step in creating a surgical plan for extensive hepatic resections of five or more segments in all patients with and without hepatic cirrhosis.
Issue Theme Liver Surgery in 2013; Guest Editors, Jean C. Emond, MD, and Michael D. Kluger, MD, MPH
Copyright (c) 2013 by Thieme Medical Publishers, Inc., 333 Seventh Avenue, New York, NY 10001, USA. Tel: +1(212) 584-4662.
Dol http://dx.doi.org/ $10.1055 / \mathrm{s}-0033-1351777$. ISSN 0272-8087. 


\section{Preoperative Assessment of Residual Hepatic Function}

\section{Estimation of Residual Liver Volume}

Dual-phase computed tomography (CT) or magnetic resonance imaging (MRI) are the radiologic techniques of choice to assess tumor location and size. The aim is to evaluate if a curative resection is possible with an acceptable low risk of postoperative liver failure. Computer-assisted volumetry of the entire liver and the liver remnant after "virtual resection" allow the calculation of either the ratio of remnant liver volume to total liver volume (RLV/TLV ratio) or the ratio of remnant liver to body volume (RLV/BW ratio). Radiologic assessment of liver size is preferable over formulas that estimate liver size. There is good general correlation between estimated and measured liver size, ${ }^{1}$ but in individual cases estimation of liver size may be erroneous depending on liver size. $^{2}$

There is no consensus if RLV/BW or RLV/TLV ratios have a better ability to predict postoperative liver failure. Traditionally a RLV/TLV ratio above 20 to $25 \%$ is considered acceptable for tumor resections. The margin of safety needs to be much higher for living liver donation than for therapeutic liver resections. For living liver donation, conventionally RLV/BW ratios are used and a ratio $>0.6$ is considered safe to avoid postoperative small for size syndrome. ${ }^{3} \mathrm{RLV} / \mathrm{BW}$ ratios are now also used for liver resections. Truant et al reported in 2004 that resections of more than $80 \%$ of the total liver volume did not increase the 3-month morbidity; however, patients who had less than $0.5 \%$ RLV/BW ratio had a significantly higher incidence of complications and mortality. ${ }^{4}$

This was a small retrospective study of only 31 patients; more recent studies have found a close correlation between RLV/BW and RLV/TLV ratios. Chun et al reported that a RLV/ BW ratio of $\leq 0.4$ or a $R L V / T L V$ ratio of $\leq 20 \%$ is a safe threshold to prevent postoperative liver failure in noncirrhotic patients. $^{5}$

Patients whose disease is more extensive may benefit from preoperative chemoembolization or radiofrequency ablation to shrink the tumor(s), staged surgery for multilobar lesions, or preoperative portal vein embolization to increase the volume of the future liver remnant to achieve safe resection of otherwise unresectable tumors. ${ }^{6}$

\section{Assessment of Operative Candidacy of Patients with Hepatic Cirrhosis}

Hepatic cirrhosis renders the liver disproportionally sensitive to ischemic injury and even short episodes of ischemiareperfusion may cause profound dysfunction of the remnant liver.

Hepatocellular carcinoma (HCC) is common with hepatic cirrhosis and is a frequent cause of mortality. Resection can improve survival and should be performed if it can be done safely, especially if the patient is not considered a transplant candidate. Staging of HCC should include the American Joint Committee on Cancer / International Union for Cancer Control definitions.

Large tumor size is not a contraindication for resection and the ability to perform a safe resection depends on the size of the liver remnant and the ability to perform the resection without vascular occlusion techniques. There is no consensus of what are considered safe RLV/BW and RLV/TLV ratios; however, a RLV/TLV ratio $<40 \%$ is generally considered a very high risk for postoperative liver failure in cirrhotic patients. ${ }^{7}$ Patients with severe liver disease (Child C) are usually not considered candidates for liver resection at all. Patients with Child B cirrhosis will not tolerate major resections with a RLV/TLV ratio $<30 \%$. The mortality of patients with Child A cirrhosis after liver resection has been reported to be $14 \%$ compared with $1 \%$ in patients without cirrhosis. ${ }^{8}$ Multiple studies have demonstrated that the Model of Endstage Liver Disease (MELD) score is a good predictor of postoperative complications such as acute liver failure and mortality after hepatic resections. ${ }^{9-11}$ These studies indicate that patients with a MELD score $>9$ are at a substantial risk for postoperative complications and mortality and (extensive) resection should probably be avoided. Interestingly, the MELD score has not been useful in predicting morbidity or mortality risk after liver resection in patients without cirrhosis. $^{12}$

Static laboratory tests may not be able to fully appreciate the ability of the liver to prevail despite reduction in size and ischemic injury associated with liver resections. As I will discuss later in this article, conventional laboratory tests are too insensitive to detect anything but severe restrictions in liver function. "Liver reserve" may be better assessed using dynamic liver function tests that are described in more detail below. Indocyanine green (ICG) clearance has been recognized as a useful test to assess the risk of liver resections for many years now. For example, Hummings et al. measured ICG clearance in 22 patients undergoing liver resection in 1992. Preoperative ICG clearance was the best predictor of mortality, better than any other liver laboratory test. ${ }^{13}$ Multiple newer studies also confirmed the utility of ICG clearance as a risk assessment tool. ${ }^{14-16}$ This has led to the development of an algorithm that includes total serum bilirubin levels as well as results from preoperative ICG clearance to aid in the decision about the extent of resection in patients with hepatic cirrhosis. $^{14}$

Other dynamic function tests, for example, the monoethylglycinexylidide (MEGX-) test ${ }^{17}$ or hippurate ratio, ${ }^{18}$ have also been tested as risk-stratification tools for hepatic resections with similar good results. We will describe these in more detail below.

Algorithms that include conventional and dynamic liver function tests can only guide decision making and are not intended to replace clinical decisions by experienced practitioners. Major resections even with total vascular occlusion are possible in patients with mild cirrhosis in the hands of experienced surgeons, hepatologists, and anesthesiologists. ${ }^{19}$

\section{Evaluation of Liver Function}

\section{Conventional Liver Function Tests}

Conventional liver function tests can be divided into tests that reflect liver function and those reflecting liver injury. Liver function can be further divided into metabolic and synthetic 
function. Metabolic function comprises pathways that include enzymes of the cytochrome family (phase I) and glucuronidation pathways (phase II). Conventional liver function tests, such as the measurement of total bilirubin, reflect phase II metabolism. However, phase II metabolism is rather robust and insensitive to ischemic injury. Therefore, total bilirubin levels may overestimate the functional reserve of the liver and remain normal even when phase I metabolism is already substantially impaired. As a consequence, the early stages of liver dysfunction are often underdiagnosed when only phase II metabolism (e.g., total bilirubin) is taken into account.

The main synthetic functions that are commonly assessed with laboratory tests are protein synthesis and synthesis of coagulation factors. Impaired protein synthesis affects albumin concentrations and is therefore included in the ChildTurcotte score. However, albumin levels are rarely specific and sensitive enough to detect minor and intermediate compromised function. Fluid retention and protein loss with ascites may further decrease albumin levels; therefore, these may be not proportional to the decrease in synthetic function only.

Impaired synthesis of coagulation factors may result in an abnormal coagulation test, such as an increase of the international normalized ratio (INR). But liver disease affects not only the synthesis of procoagulant factors, but also anticoagulant factors such as protein Cor protein S. The balance of proand anticoagulant factors may therefore be preserved (but much less stable) or tipped toward hypercoagulability even when the INR is substantially increased.

Transaminases reflect hepatic injury; they are intracellular and intramitochondrial enzymes that are released when cell death occurs. Transaminases may reflect the degree of injury, but not necessarily impairment of function. Transaminases only increase with acute injury, but not with chronic disease; therefore, they are similar to troponin I in the presence of myocardial dysfunction. They are useful to detect acute organ injury, but not impairments of function.

These limitations of conventional liver function tests compromise their utility to assess hepatic reserve prior to liver resection. Conventional liver function becomes pathologic only late during disease progression. Therefore, patients usually do not tolerate any resections and prolonged hepatic ischemia when conventional tests are increased and other, more sensitive liver function tests may be better suited to evaluate preoperative hepatic reserve.

\section{Dynamic Liver Function Tests}

Dynamic liver function tests directly measure hepatic metabolism, usually of phase I oxidative pathways. These pathways are more sensitive to ischemia than phase II glucuronidation and will be abnormal even with mild forms of liver disease. Hence, these tests are more sensitive to detect even minor impairments of liver function. Dynamic liver function tests have the further advantage of assessing the metabolic reserve at the time of measurement, instead of relying on metabolites that may have accumulated and therefore allow a real-time assessment of function. - Fig. 1 depicts different static and dynamic liver function tests. This review will be limited to the most commonly studied tests, indocyanine green clearance, the monoethylglycinexylidide (MEGX) test, and sorbitol clearance.

\section{Indocyanine Green Clearance}

Indocyanine green (ICG) is a fluorescent dye that if given intravenously is eliminated through the bile within a few

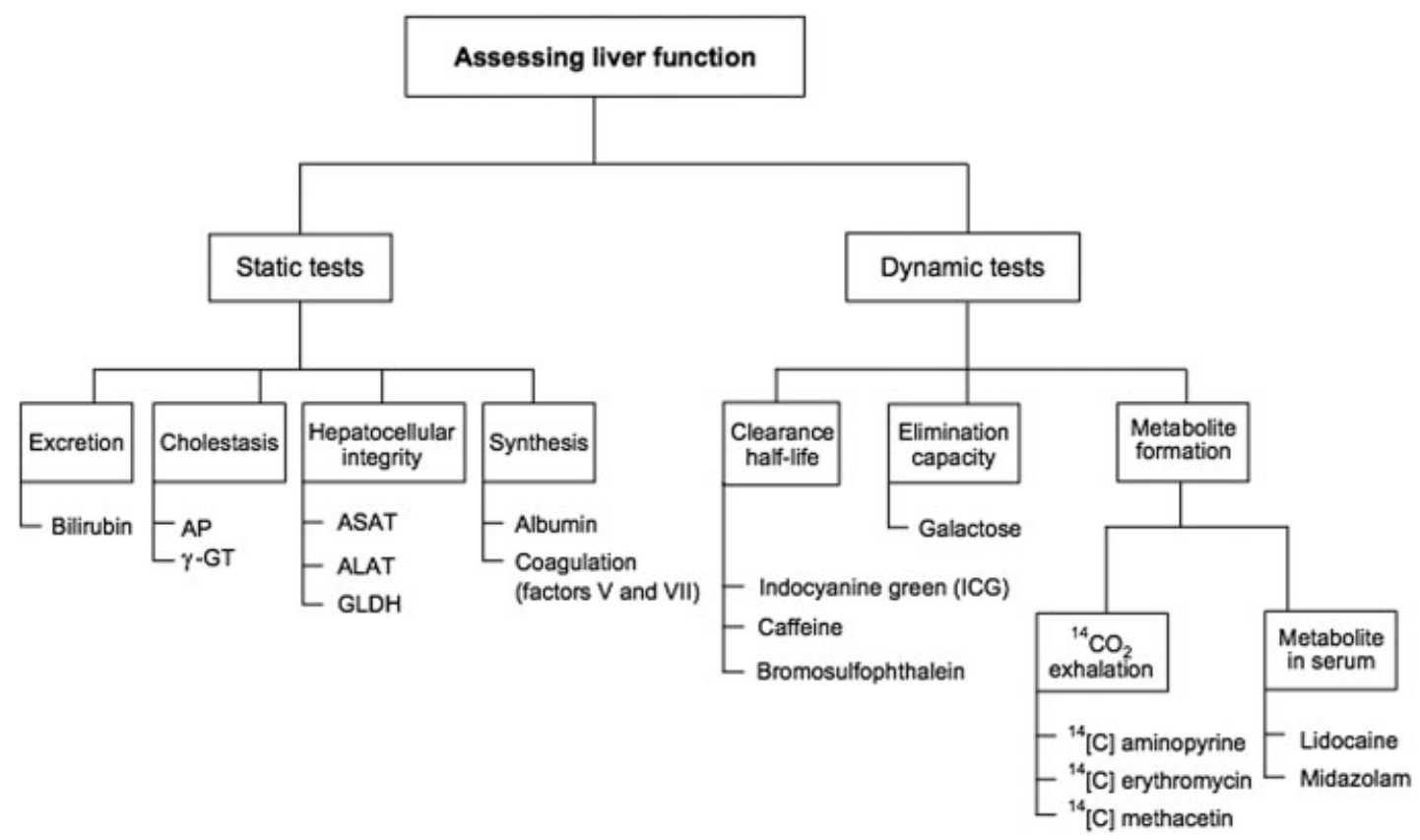

Fig. 1 Static and dynamic tests to assess liver function. AP, alkaline phosphatase; $y$-GT, gamma-glutamyl transpeptidase; ASAT, aspartate aminotransferase; ALAT, alanine aminotransferase; GLDH, glutamate dehydrogenase. (With permission from Sakka SG. Assessing liver function. Curr Opin Crit Care 2007;13(2):207-214.) 
minutes in healthy subjects and does not undergo enterohepatic recirculation. Elimination $\left(700 \mathrm{~mL} / \mathrm{min} / \mathrm{m}^{2}\right)$ is dependent on hepatocyte function, liver blood flow, and intact bile secretion and can be expressed by the percentage of ICG eliminated per minute (ICG plasma disappearance rate [ICGPDR]). Normal ICG-PDR is over $18 \%$ / min and is usually measured over 15 minutes after injection of ICG. Indocyanine green absorbs infrared light at a frequency close to the absorption spectrum of deoxygenated blood; oxygen saturation measured by pulse oximetry decreases falsely for minutes after an intravenous bolus of ICG. A transcutaneous sensor similar to a conventional pulse oximeter, but with an infrared light source at the absorption spectrum of ICG (805 and $940 \mathrm{~nm}$ ) may be used for noninvasive transcutaneous ICG clearance and has correlated very well with sequential plasma measurements of ICG. ${ }^{20}$ Multiple studies confirmed the ability of ICG clearance to predict liver failure after hepatic resection. Hemming et al found that ICG-PDR was the best predictor of 30-day mortality after liver resection and an ICGPDR. ${ }^{13}$

Furthermore, indocyanine green is a good predictor of graft loss or death within 30 days after liver transplantation. ${ }^{21}$ An ICG-PDR $<9.8 \%$ on day 7 predicted graft loss or death with a sensitivity of $75.0 \%$ and a specificity of $72.6 \%$. As ICG-PDR depends on hepatic blood flow it has also been used to detect vascular complications during liver transplantation. Renn et al reported a case of portal vein and hepatic artery occlusion after liver transplantation that was only detected by a sudden decrease in ICG-PDR. Mandell et $\mathrm{al}^{22}$ describe a similar case in which a decrease of ICG-PDR led to the diagnosis of portal vein occlusion and allowed a rapid surgical correction and full recovery of graft function.

Indocyanine green plasma disappearance rate is also an excellent predictor of outcome and survival in intensive care unit (ICU) patients or patients with sepsis ${ }^{23}$ an ICG-PDR $<8 \%$ is associated with a very high mortality. Surprisingly, ICG-PDR in this general ICU population was a better predictor of mortality than complex scoring systems such as the APACHE II (Acute Physiology and Chronic Health Evaluation II) or SAPS II (Simplified Acute Physiology Score II) scores.

Indocyanine green clearance is affected by biliary obstruction and can overestimate hepatic dysfunction when cholestasis is present. Indocyanine green has very few adverse effects, but should be avoided in patients with iodine allergies. It has been approved by the Food and Drug Administration to measure liver function, but the noninvasive, transcutaneous measurement of ICG clearance (LIMON, Pulsion Medical Systems AG, Feldkirchen, Germany) has not been approved.

\section{Monoethylglycinexylidide (MEGX) Test}

Lidocaine is an amide local anesthetic that has a high hepatic extraction ratio (0.53) and is then rapidly metabolized by the cytochrome P450 system through oxidative $N$-dealkylation. Its main metabolite is monoethylglycinexylidide (MEGX) can be easily measured in the plasma. Plasma MEGX levels can be determined before and then 15 and 30 minutes after a bolus injection of intravenous lidocaine. The rate of lidocaine metabolism depends on hepatic blood flow and the activity of the cytochrome P-450 system, specifically the CYP 3 A4 and CYP $1 \mathrm{~A} 2$ isoenzymes. ${ }^{24,25}$ Erythromycin and ketoconazole inhibit the CYP 3A4 isoenzyme and can therefore affect the MEGX test adversely. Oxidative phase I metabolism by cytochrome $\mathrm{P} 450$ is more sensitive to ischemic insults than phase II glucuronidation and is therefore better suited to assess hepatic reserve. The MEGX is a good predictor of survival in patients with liver disease. ${ }^{26-28}$ Other studies have successfully used the MEGX test to assess cadaveric liver donor quality $^{29,30}$ or posttransplant graft function. ${ }^{31}$

Similar to ICG clearance, MEGX measurements are also highly predictive of survival in general ICU populations. On day 4 after ICU admission, median MEGX levels were significantly lower in nonsurvivors than in survivors ( $23 \mu \mathrm{g} / \mathrm{L}$ vs. 53 $\mu \mathrm{g} / \mathrm{L}, \boldsymbol{p}<0.01){ }^{32}$ These results emphasize that hepatic dysfunction is much more common in critically ill patients and not readily detected with conventional liver function tests such as total bilirubin measurements. Despite this, MEGX testing is not routinely used in clinical practice.

\section{Sorbitol Clearance}

D-sorbitol is a nontoxic sugar with a very high hepatic extraction ratio (0.93) and is rapidly metabolized by the fructose pathway in the liver. ${ }^{33}$ Because of the high hepatic extraction of D-sorbitol, its elimination correlates well with hepatic blood flow. In hepatic cirrhosis, total hepatic blood flow is not necessarily decreased, but the amount of effective blood flow, i.e., the fraction of total blood flow that gets in contact with hepatic sinusoids declines. A larger proportion of blood bypasses constricted sinusoids through trans- and extrahepatic shunts; therefore, it does not participate in the hepatic metabolism. ${ }^{34}$

D-sorbitol elimination (and to some degree ICG clearance) is therefore likely a reflection of "functional" blood flow and not total hepatic blood flow. D-sorbitol has a higher extraction ratio than ICG (0.93 and 0.58 , respectively) and is therefore less dependent on metabolic reserve of the liver and may more accurately estimate functional liver flow. There are few human studies of D-sorbitol elimination; more research is required to assess its clinical utility for liver resection and transplantation.

\section{Preoperative Risk Assessment for Nonhepatic Disease}

\section{Cardiac and Coronary Disease}

The most commonly used algorithm to aid in the decision about preoperative cardiac testing are the guidelines of the American College of Cardiology / American Heart Association on perioperative cardiovascular evaluation and care for noncardiac surgery (revised in 2007). ${ }^{35}$ This algorithm considers any intraperitoneal surgery (which includes major hepatic resections) as intermediate risk surgery (only vascular surgery is considered high risk). Patients with poor $(<4$ metabolic equivalents of task [METs]) or unknown functional capacity who have three or more clinical risk factors for cardiac events should undergo noninvasive testing for cardiac disease. Patients with 1 to 2 clinical risk factors can 
undergo noninvasive testing for cardiac disease especially if this may change the perioperative management. Clinical risk factors include a history of ischemic heart disease, compensated or prior heart failure or cerebrovascular disease, diabetes mellitus, or renal insufficiency.

Patients with active cardiac conditions such as unstable coronary syndromes, decompensated heart failure (New York Heart Association [NYHA] functional class IV, worsening or new-onset heart failure), significant arrhythmias, or severe valvular disease should undergo testing prior to surgery in all cases except emergencies independent of ability to exercise.

The use of this algorithm may not be feasible when patients have rapidly growing or large tumors. Stress test, coronary angiography, and possible intervention either by percutaneous intervention or coronary bypass grafting may take too long and cause the tumor to be unresectable, hence taking away the patient's chance for cure. In these cases, surgery should proceed as long as the patient fully understands the increased risk for cardiac events and agrees with this plan.

Multiple other risk-assessment tools have been developed. The Revised Cardiac Risk Index ${ }^{36}$ is probably the most widely used tool that is simple and easily applied with few questions. One point each is given for high-risk surgery (including intraperitoneal), a history of ischemic heart disease, congestive heart failure or cerebrovascular disease, preoperative treatment with insulin, or preoperative serum creatinine $>2.0 \mathrm{mg} / \mathrm{dL}$.

The risk of major cardiac events increases with an increasing number of risk factors and is $0.4 \%$ for $0,0.9 \%$ for $1,6.6 \%$ for 2 , and $11 \%$ for 3 or more points. This simple score has good predictive power, but has not been validated for patients undergoing hepatectomies.

\section{Intraoperative Management}

\section{Anesthetic Management and Monitoring and Low Central Venous Pressure Technique}

General endotracheal anesthesia is commonly required for hepatectomies.

(Radial) arterial catheters should be placed routinely to allow beat-to-beat blood pressure monitoring and facilitate frequent blood draws. Major (but not necessarily minor) resections will also require placement of a central venous catheter that allows monitoring of central venous pressure (CVP), large volume fluid administration, and central administration of vasoactive drugs.

Central venous pressure is not an adequate representation of volume status and responsiveness. Multiple studies starting with Shoemaker's study of intravascular volume and CVP 30 years ago ${ }^{37}$ have demonstrated that there is no relationship between CVP and intravascular volume. Central venous pressure also does not predict which patients respond to a volume challenge favorably (i.e., with an increased blood pressure). ${ }^{38}$

However, during liver resections CVP monitoring may be helpful. Central venous pressure correlates with hepatic vein pressure; therefore, intraparenchymal pressure will increase with increased CVP. Many studies have demonstrated that low CVP ( $<5 \mathrm{~mm} \mathrm{Hg}$ ) is associated with decreased blood loss ${ }^{39-41}$ and perhaps even improved morbidity and mortality. ${ }^{42}$ These results have been criticized by some practitioners; two studies found no association between CVP and blood loss during living liver donation. Low CVP is not without risk; for example, low renal perfusion may exacerbate pre-existing renal insufficiency and cause acute kidney injury. This is particularly of concern if the patient has underlying hepatic cirrhosis and is dependent on a hyperdynamic state and high cardiac output to maintain (renal) perfusion pressure. Low CVP technique may furthermore affect beneficial hepatic artery flow and oxygen delivery to the liver.

In patients with no renal dysfunction and normal liver function, the low CVP technique is recommended. Reduction in blood loss and avoidance of blood transfusion facilitates resection and is likely going to improve outcome. ${ }^{43}$ Adequate fluid resuscitation after the specimen was removed should avoid any adverse sequelae in most cases. Frequently, vasopressors are temporarily required to maintain perfusion pressure when using the low CVP technique.

Low CVP technique is not indicated if total hepatic occlusion is required. Occlusion of the suprahepatic inferior vena cava (IVC) substantially decreases cardiac preload, which is not tolerated if the patient is hypovolemic. Inferior vena cava clamping will require an adequate volume status in addition to vasopressor administration.

\section{Surgical and Anesthetic Techniques to Preserve Liver Function}

Frequently, major liver resections will require vascular occlusion to reduce blood loss and optimize the surgical field. Vascular inflow occlusion is often achieved by slinging an umbilical tape around the porta hepatis and then tightening it. This technique is called the Pringle maneuver and disrupts portal venous and hepatic arterial blood flow to the liver. The Pringle maneuver causes ischemic injury to the liver and can therefore only be applied for a limited time. This is particularly important if the liver has underlying disease such as hepatic cirrhosis. Cirrhotic livers are extraordinarily sensitive to ischemic injury. Application of the Pringle maneuver in cirrhotic patients causes significantly higher postoperative elevations of conventional liver function tests compared with noncirrhotic patients. ${ }^{44}$ If possible, the Pringle maneuver should be avoided or its duration minimized in patients with hepatic cirrhosis.

Cholestatic livers are similarly sensitive to ischemic insults as cirrhotic livers. Reversible causes of cholestatic disease should be treated prior to major liver resections. ${ }^{45}$ Stenting and drainage of extrahepatic biliary obstructions and then delay of surgery until resolution of cholestasis can greatly reduce the risk of postoperative liver failure. ${ }^{46}$

Earlier studies in animals ${ }^{47}$ and humans ${ }^{48,49}$ using ischemic preconditioning to ameliorate hepatic injury had been quite promising. For example, Clavien et al randomized 100 patients to 10 minutes of hepatic ischemia followed by 10 minutes reperfusion or no ischemic preconditioning. All patients then underwent major hepatic resection with at 
least 30-minute ischemic time. The authors found that patients who had undergone ischemic preconditioning had lower postoperative transaminase levels. This beneficial effect was particularly noticeable in young patients. However, a more recent meta-analysis found a similar decrease in transaminase levels, but no improvement of mortality, liver function, or other morbidity between the groups. ${ }^{50}$ These results were lately confirmed for patients undergoing liver resection with total vascular exclusion (TVE). Jeon et al found no difference in outcome or laboratory markers in patients who received ischemic preconditioning when undergoing major liver resection with TVE compared with patients without ischemic preconditioning. ${ }^{51}$ Due to this absence of convincing evidence, we cannot recommend the routine use of ischemic preconditioning.

Selective vascular inflow occlusion may be used by occluding only the right or left branches of the portal vein and hepatic artery. This technique will require a more complex dissection of the porta hepatis, but results in decreased injury to the remaining lobe of the liver and may be a very suitable technique to avoid ischemic injury in patients with preexisting liver dysfunction and cirrhosis.

Total vascular exclusion comprises temporary occlusion of the porta hepatis (e.g., using the Pringle maneuver) and clamping of the IVC both above and below the liver. This results in substantial reduction of right ventricular preload and is usually not tolerated when a low CVP technique is used. Fluid loading prior to IVC clamping is mandatory; additional vasopressors are often required to maintain blood pressure. This is particularly true in patients with underlying liver disease who need a hyperdynamic circulation to compensate for low systemic vascular resistance. Close communication about the surgical plan between the anesthesiologist and surgeon is essential to avoid potentially catastrophic events.

\section{Postoperative Management}

\section{Routine Postoperative Management}

The perioperative mortality for patients undergoing hepatic resections has vastly improved over the last few decades, and now is $5 \%$ or less in high volume centers. The most important factors affecting perioperative mortality are blood loss, the amount of liver resected, and the functional reserve of the remaining liver. ${ }^{52}$ Consideration to these factors during the surgery is essential to guide postoperative management and to achieve favorable outcomes.

In the absence of underlying hepatic dysfunction or significant comorbidities, patients undergoing simple partial hepatectomy may be safely managed in a stepdown unit during the first postoperative day. Because patients with baseline hepatic dysfunction are at increased risk of complications in the immediate postoperative period, we recommend ICUlevel care, invasive blood pressure monitoring, and a urinary catheter to measure urine output. Continuous blood pressure monitoring with a radial arterial line is standard, and routine use of a femoral arterial line is usually not required unless an extensive hepatic resection with total vascular exclusion is expected. Total vascular occlusion, including clamping of the vena cava, causes profound hypotension requiring high-dose vasopressors. This may dampen the tracing of the radial artery catheter; in this situation, a femoral arterial line will continue to provide reliable arterial blood pressure data.

Central line access is usually recommended for major resections to allow rapid administration of fluid, blood, and vasoactive drugs if necessary.

Most patients will not require postoperative ventilatory support; if the patient arrives intubated in the ICU, extubation can proceed rapidly as long as there are no major complications. Large fluid and transfusion requirements, bleeding, and hemodynamic instability may preclude extubation. The patients may require longer ventilatory support if the development of liver failure due to a small liver remnant is suspected. Liver failure will result in decreased lactate metabolism and metabolic acidosis that will require the patient to hyperventilate to maintain a normal $\mathrm{pH}$. It will be safer to support the patient with a ventilator during this time to avoid exhaustion until the metabolic acidosis is resolving.

\section{Fluids and Electrolytes}

Patients with cirrhosis have high circulating blood volume requirements and after large abdominal surgery are susceptible to fluid shifts, reaccumulation of ascites, and episodes of hypotension. Colloid fluid administration is effective for bolus resuscitation to rapidly restore intravascular volume. Hypotension refractory to fluid administration should be treated with vasopressors (norepinephrine \pm vasopressin) and the cause for hypotension needs to be swiftly investigated. Hypovolemia due to underresuscitation or bleeding are common and may be exaggerated with underlying cirrhotic vasodilation.

An understanding of the intraoperative events, estimated blood loss, and total fluid volume given including blood products will help guide the initial management. Close observation should be paid to achieve adequate end organ perfusion with a goal urine output of $>0.5 \mathrm{~mL} / \mathrm{kg} / \mathrm{h}$.

Acute kidney injury is common after major hepatic resection with a reported incidence of up to $15 \% .{ }^{53}$ In patients with hepatic cirrhosis, acute kidney injury is often confounded by prerenal azotemia and hepatorenal syndrome (HRS). Hepatorenal syndrome occurs with advanced cirrhosis and results from peripheral and splanchnic vasodilation, leading to decreased renal perfusion, increased sodium reabsorption, and total body volume overload. Inadequate resuscitation and infection predispose to HRS; albumin and vasopressors administration and antibiotics may facilitate clinical improvement. $^{54}$

Drains placed in the abdomen should be observed for signs of hemorrhage or bile leak. The character of the fluid draining should be corroborated with the patient's clinical picture, keeping in mind that drains may have shifted or clogged. The absence of significant drainage does not rule out the presence of bleeding or bile leak. Bile leaks are common and may occur in up to $20 \%$ of hepatectomies.

As fluid shifts between vascular compartments, occasionally the judicious use of diuretics can help prevent volume 
overload beyond postoperative day 2; though care should be taken to assess the patient's complete clinical picture. Overdiuresis and low circulating intravascular volume can have profound consequences as mentioned above.

Diligent replacement of electrolytes is prudent because nearly all posthepatectomy patients will have some degree of electrolyte abnormalities in the postoperative period. Hypophosphatemia occurs frequently and aggressive repletion is appropriate to prevent deficient energy metabolism manifesting as cardiac or respiratory dysfunction. ${ }^{55}$

\section{Postoperative Hepatic Function}

The risk of postoperative hepatic dysfunction in patients with normal underlying liver parenchyma is low as long as sufficient hepatic volume remains. After major resections vigilance and close postoperative monitoring of liver function is essential to identify patients at risk for liver failure. Initial laboratory studies should include a complete blood count, basic metabolic panel, INR, partial thromboplastin time (PTT), hepatic function tests, and lactate level. The INR and lactate levels should be measured frequently; these are the fastest and most sensitive and specific of conventional laboratory markers. Transaminases will initially increase from intraoperative hepatic manipulation, and total serum bilirubin levels may require days to increase. Decreasing arterial lactate levels will help assess adequate hepatic function. Additional signs that the liver is sufficiently functioning include increased blood glucose levels from hepatic gluconeogenesis.

For patients undergoing hepatic artery or portal vein reconstruction, an abdominal Doppler should be obtained to ensure adequate flow and normal velocities. Portal vein thrombosis may present as large volume ascites, encephalopathy, and liver failure and needs to be addressed rapidly. Venous thromboembolism prophylaxis should be weighed against the risk of bleeding. Liver dysfunction is associated with a decrease in pro- and anticoagulant factors and thrombin formation may be normal or even increased even in the presence of increased coagulation parameters. ${ }^{56}$ Low-molecular-weight heparin or unfractionated heparin should be administered if there is no risk of significant bleeding. 57

Posthepatectomy liver failure (PHLF) has been defined by a 2011 international consensus as the presence of elevated INR and hyperbilirubinemia on postoperative day 5. It is further subdivided into three clinical grades: (1) biological liver failure requiring no change in management, (2) deviation from regular course requiring no invasive therapy, and (3) invasive treatment required. To date, no studies have shown improved survival when treating PHLF, and the only definitive treatment remains orthotopic liver transplantation. Supportive care focuses on identifying and treating the underlying etiology. Institution of antibiotic therapy, identification of vascular compromise or biliary leak, and appropriate nutritional supplementation (30\% more in stressed states) are essential elements of management. Encephalopathy should be treated, for example, with lactulose, and mannitol can be utilized to reduce intracranial hypertension. Clinically significant coagulopathy from thrombocytopenia or elevated INR resulting in bleeding will require administration of clotting factors and blood products. However, we caution against administering clotting factors only to treat elevated laboratory tests in the absence of clinical significant bleeding or prior to invasive procedures. ${ }^{58}$

\section{The Patient with Liver Disease Undergoing Nonhepatic Surgery}

Determination of which patients are at risk of liver dysfunction after surgery is not clearly defined. Of note, the available literature of surgical risk in patients with cirrhosis is limited to retrospective investigations. Furthermore, these studies typically involve patients with only mild hepatic dysfunction, limiting their applicability to those most at risk. Clearly, elective surgery is less common for patients who simply will not survive without urgent liver transplantation. However, in those less-acute patients with liver disease, efforts to clearly define operative candidacy have be challenging.

The Child-Turcotte-Pugh (CTP) class can be quickly calculated at the bedside and is the metric often used in the clinical studies, correlating well with mortality. The CTP scoring system is based on the patient's serum bilirubin, albumin, prothrombin time, as well as the severity of ascites and encephalopathy. ${ }^{59}$ The CTP class is often criticized based on the subjective nature for two of its parameters (ascites and encephalopathy) and its broad variation of hepatic function within each class, especially within class B. Nonetheless, mortality rates are predictable following general surgery (CTP-A $10 \%$, СТP-B 30\%, CTP-C 76-82\%). ${ }^{59}$ The CTP-B or $\mathrm{CTP}-\mathrm{C}$ patients are not candidates for elective major surgery. The CTP-A patients with significant portal hypertension characterized by thrombocytopenia (i.e., platelets $<100,000$ ), esophageal varices, or elevated portal pressures by invasive monitoring are rarely candidates for elective major surgery, and definitely not candidates for major liver resections.

The model for end-stage liver disease (MELD) was first developed to determine survival after transjugular intrahepatic portocaval shunt (TIPS) procedures. However, in 2002 MELD was identified as a reliable index that could predict short-term mortality rates of liver transplantation candidates. ${ }^{60}$ The MELD score is calculated based on serum bilirubin, serum creatinine, and international normalized ratio. The MELD is more objective than the CTP score and is a good predictor of 30-day mortality in patients with cirrhosis undergoing nontransplant surgery. ${ }^{61}$ The postoperative mortality rate in nontransplant patients rises in a linear fashion $1 \%$ for each increase in MELD point below 20 and 2\% for MELDs above $20{ }^{61}$ Furthermore, several authors have demonstrated that MELD scores above 14 predict a poor outcome after intraabdominal surgery. ${ }^{62-64}$ Some have also demonstrated that a MELD > 14 is comparable to CTP class C and that MELD scores can correctly predict poor outcomes better the CTP score (77 vs. $23 \%){ }^{64}$ 


\section{Summary}

Patients with underlying liver disease undergoing hepatic resection face substantial risk of postoperative liver failure. Precise assessment of preoperative liver function and reserve, the extent of resection, and other comorbidities is essential to be able to decide if the patient is a candidate for surgery. The extent of resection can be evaluated using computed tomography or magnetic resonance imaging scans and computer simulations that allow virtual resection prior to surgery.

Comorbidities such as cardiac disease should be assessed according to standard guidelines for no-cardiac surgery such as the American Society of Anesthesiologists / American Heart Association guidelines. Conventional liver function tests are frequently too insensitive to assess liver reserve. Dynamic liver function tests such as ICG clearance may be better suited to detect patients with limited hepatic reserve who may not tolerate major resections. Intraoperative management should aim to reduce further injury to the diseased liver by avoiding hepatic ischemia, volume overload or bleeding. Algorithms may help assess the risk of major resections in patients with underlying liver disease. However, in complex cases, the decision about operability should be made by a team of experienced surgeons, hepatologists, and anesthesiologists.

\section{References}

1 Pomposelli JJ, Tongyoo A, Wald C, Pomfret EA. Variability of standard liver volume estimation versus software-assisted total liver volume measurement. Liver Transpl 2012;18(9):1083-1092

2 Siriwardana RC, Chan SC, Chok KS, Lo CM, Fan ST. Effects of the liver volume and donor steatosis on errors in the estimated standard liver volume. Liver Transpl 2011;17(12):1437-1442

3 Tucker ON, Heaton N. The 'small for size' liver syndrome. Curr Opin Crit Care 2005;11(2):150-155

4 Truant S, Oberlin O, Sergent G, et al. Remnant liver volume to body weight ratio $>$ or $=0.5 \%$ : A new cut-off to estimate postoperative risks after extended resection in noncirrhotic liver. J Am Coll Surg 2007;204(1):22-33

5 Chun YS, Ribero D, Abdalla EK, et al. Comparison of two methods of future liver remnant volume measurement. J Gastrointest Surg 2008;12(1):123-128

6 Vauthey JN, Zorzi D, Pawlik TM. Making unresectable hepatic colorectal metastases resectable-does it work? Semin Oncol 2005;32(6, Suppl 9):S118-S122

7 Vauthey JN, Dixon E, Abdalla EK, et al; American Hepato-Pancreato-Biliary Association; Society of Surgical Oncology; Society for Surgery of the Alimentary Tract. Pretreatment assessment of hepatocellular carcinoma: expert consensus statement. HPB (Oxford) 2010;12(5):289-299

8 Vauthey JN, Klimstra D, Franceschi D, et al. Factors affecting longterm outcome after hepatic resection for hepatocellular carcinoma. Am J Surg 1995;169(1):28-34, discussion 34-35

9 Teh SH, Christein J, Donohue J, et al. Hepatic resection of hepatocellular carcinoma in patients with cirrhosis: Model of End-Stage Liver Disease (MELD) score predicts perioperative mortality. J Gastrointest Surg 2005;9(9):1207-1215, discussion 1215

10 Cucchetti A, Ercolani G, Vivarelli M, et al. Impact of model for endstage liver disease (MELD) score on prognosis after hepatectomy for hepatocellular carcinoma on cirrhosis. Liver Transpl 2006;12 (6):966-971
11 Delis SG, Bakoyiannis A, Biliatis I, Athanassiou K, Tassopoulos N, Dervenis C. Model for end-stage liver disease (MELD) score, as a prognostic factor for post-operative morbidity and mortality in cirrhotic patients, undergoing hepatectomy for hepatocellular carcinoma. HPB (Oxford) 2009;11(4):351-357

12 Teh SH, Sheppard BC, Schwartz J, Orloff SL. Model for end-stage liver disease score fails to predict perioperative outcome after hepatic resection for hepatocellular carcinoma in patients without cirrhosis. Am J Surg 2008;195(5):697-701

13 Hemming AW, Scudamore CH, Shackleton CR, Pudek M, Erb SR. Indocyanine green clearance as a predictor of successful hepatic resection in cirrhotic patients. Am J Surg 1992;163(5):515-518

14 Seyama Y, Kokudo N. Assessment of liver function for safe hepatic resection. Hepatol Res 2009;39(2):107-116

15 Hashimoto M, Watanabe G. Hepatic parenchymal cell volume and the indocyanine green tolerance test. J Surg Res 2000;92(2): 222-227

16 Scheingraber S, Richter S, Igna D, Flesch S, Kopp B, Schilling MK. Indocyanine green disappearance rate is the most useful marker for liver resection. Hepatogastroenterology 2008;55(85): 1394-1399

17 Lorf T, Schnitzbauer AA, Schaefers SK, et al. Prognostic value of the monoethylglycinexylidide (MEGX)-test prior to liver resection. Hepatogastroenterology 2008;55(82-83):539-543

18 Hemming AW, Gallinger S, Greig PD, et al. The hippurate ratio as an indicator of functional hepatic reserve for resection of hepatocellular carcinoma in cirrhotic patients. J Gastrointest Surg 2001;5 (3):316-321

19 Emond JC, Kelley SD, Heffron TG, Nakagawa T, Roberts JP, Lim RC Jr. Surgical and anesthetic management of patients undergoing major hepatectomy using total vascular exclusion. Liver Transpl Surg 1996;2(2):91-98

20 Sakka SG, van Hout N. Relation between indocyanine green (ICG) plasma disappearance rate and ICG blood clearance in critically ill patients. Intensive Care Med 2006;32(5):766-769

21 Schneider L, Spiegel M, Latanowicz S, et al. Noninvasive indocyanine green plasma disappearance rate predicts early complications, graft failure or death after liver transplantation. Hepatobiliary Pancreat Dis Int 2011;10(4):362-368

22 Mandell MS, Wachs M, Niemann CU, Henthorn TK. Elimination of indocyanine green in the perioperative evaluation of donor liver function. Anesth Analg 2002;95(5):1182-1184 table of contents.

23 Sakka SG, Reinhart K, Meier-Hellmann A. Prognostic value of the indocyanine green plasma disappearance rate in critically ill patients. Chest 2002;122(5):1715-1720

24 Bargetzi MJ, Aoyama T, Gonzalez FJ, Meyer UA. Lidocaine metabolism in human liver microsomes by cytochrome P450IIIA4. Clin Pharmacol Ther 1989;46(5):521-527

25 Wang JS, Backman JT, Wen X, Taavitsainen P, Neuvonen PJ, Kivistö KT. Fluvoxamine is a more potent inhibitor of lidocaine metabolism than ketoconazole and erythromycin in vitro. Pharmacol Toxicol 1999;85(5):201-205

26 Shiffman ML, Luketic VA, Sanyal AJ, et al. Hepatic lidocaine metabolism and liver histology in patients with chronic hepatitis and cirrhosis. Hepatology 1994;19(4):933-940

27 Elin RJ, Fried MW, Sampson M, Ruddel M, Kleiner DE, DiBisceglie AM. Assessment of monoethylglycinexylidide as measure of liver function for patients with chronic viral hepatitis. Clin Chem 1997;43(10):1952-1957

28 Meyer-Wyss B, Renner E, Luo H, Scholer A. Assessment of lidocaine metabolite formation in comparison with other quantitative liver function tests. J Hepatol 1993;19(1):133-139

29 Zotz RB, von Schönfeld J, Erhard J, et al. Value of an extended monoethylglycinexylidide formation test and other dynamic liver function tests in liver transplant donors. Transplantation 1997;63 (4):538-541 
30 Olinga P, Maring JK, Groothuis GM, et al. Value of the in vitro or in vivo monoethylglycinexylidide test for predicting liver graft function. Transplantation 1997;64(1):60-65

31 Schütz E, Luy-Kaltefleiter M, Kaltefleiter M, et al. The value of serial determination of MEGX and hyaluronic acid early after orthotopic liver transplantation. Eur J Clin Invest 1996;26(10):907-916

32 Schröter J, Wandel C, Böhrer H, Schmidt H, Böttiger BW, Martin E. Lignocaine metabolite formation: an indicator for liver dysfunction and predictor of survival in surgical intensive care patients. Anaesthesia 1995;50(10):850-854

33 Blakley RL. The metabolism and antiketogenic effects of sorbitol; sorbitol dehydrogenase. Biochem J 1951;49(3):257-271

34 Molino G, Avagnina P, Belforte G, Bircher J. Assessment of the hepatic circulation in humans: new concepts based on evidence derived from a D-sorbitol clearance method. J Lab Clin Med 1998;131(5):393-405

35 Fleisher LA, Beckman JA, Brown KA, et al. ACC/AHA 2007 Guidelines on Perioperative Cardiovascular Evaluation and Care for Noncardiac Surgery: Executive Summary: A Report of the American College of Cardiology/American Heart Association Task Force on Practice Guidelines (Writing Committee to Revise the 2002 Guidelines on Perioperative Cardiovascular Evaluation for Noncardiac Surgery) Developed in Collaboration With the American Society of Echocardiography, American Society of Nuclear Cardiology, Heart Rhythm Society, Society of Cardiovascular Anesthesiologists, Society for Cardiovascular Angiography and Interventions, Society for Vascular Medicine and Biology, and Society for Vascular Surgery. J Am Coll Cardiol 2007;50(17): $1707-1732$

36 Lee TH, Marcantonio ER, Mangione CM, et al. Derivation and prospective validation of a simple index for prediction of cardiac risk of major noncardiac surgery. Circulation 1999;100(10):10431049

37 Shippy CR, Appel PL, Shoemaker WC. Reliability of clinical monitoring to assess blood volume in critically ill patients. Crit Care Med 1984;12(2):107-112

38 Marik PE, Baram M, Vahid B. Does central venous pressure predict fluid responsiveness? A systematic review of the literature and the tale of seven mares. Chest 2008;134(1):172-178

39 Smyrniotis V, Kostopanagiotou G, Theodoraki K, Tsantoulas D, Contis JC. The role of central venous pressure and type of vascular control in blood loss during major liver resections. Am J Surg 2004;187(3):398-402

40 Jones RM, Moulton CE, Hardy KJ. Central venous pressure and its effect on blood loss during liver resection. Br J Surg 1998;85(8): 1058-1060

41 Wang WD, Liang LJ, Huang XQ Yin XY. Low central venous pressure reduces blood loss in hepatectomy. World J Gastroenterol 2006;12 (6):935-939

42 Chen H, Merchant NB, Didolkar MS. Hepatic resection using intermittent vascular inflow occlusion and low central venous pressure anesthesia improves morbidity and mortality. J Gastrointest Surg 2000;4(2):162-167

43 Gozzetti G, Mazziotti A, Grazi GL, et al. Liver resection without blood transfusion. Br J Surg 1995;82(8):1105-1110

44 Sugiyama Y, Ishizaki Y, Imamura H, Sugo H, Yoshimoto J, Kawasaki S. Effects of intermittent Pringle's manoeuvre on cirrhotic compared with normal liver. Br J Surg 2010;97(7):1062-1069

45 Fan ST. Methods and related drawbacks in the estimation of surgical risks in cirrhotic patients undergoing hepatectomy. Hepatogastroenterology 2002;49(43):17-20

46 Kloek JJ, Marsman HA, van Vliet AK, Gouma DJ, van Gulik TM. Biliary drainage attenuates postischemic reperfusion injury in the cholestatic rat liver. Surgery 2008;144(1):22-31
47 Peralta C, Hotter G, Closa D, Gelpí E, Bulbena O, Roselló-Catafau J. Protective effect of preconditioning on the injury associated to hepatic ischemia-reperfusion in the rat: role of nitric oxide and adenosine. Hepatology 1997;25(4):934-937

48 Clavien PA, Selzner M, Rüdiger HA, et al. A prospective randomized study in 100 consecutive patients undergoing major liver resection with versus without ischemic preconditioning. Ann Surg 2003;238(6):843-850, discussion 851-852

49 Clavien PA, Yadav S, Sindram D, Bentley RC. Protective effects of ischemic preconditioning for liver resection performed under inflow occlusion in humans. Ann Surg 2000;232(2):155-162

50 Gurusamy KS, Kumar Y, Pamecha V, Sharma D, Davidson BR. Ischaemic pre-conditioning for elective liver resections performed under vascular occlusion. Cochrane Database Syst Rev 2009;(1): CD007629

51 Jeon J, Watkins A, Wagener G, et al. Complex hepatectomy under total vascular exclusion of the liver: impact of ischemic preconditioning on clinical outcomes. World J Surg 2013;37(4): 838-846

52 Jarnagin WR, Gonen M, Fong Y, et al. Improvement in perioperative outcome after hepatic resection: analysis of 1,803 consecutive cases over the past decade. Ann Surg 2002;236(4):397-406, discussion 406-407

53 Saner F. Kidney failure following liver resection. Transplant Proc 2008;40(4):1221-1224

54 Halimi C, Bonnard P, Bernard B, et al. Effect of terlipressin (Glypressin) on hepatorenal syndrome in cirrhotic patients: results of a multicentre pilot study. Eur J Gastroenterol Hepatol 2002;14(2): 153-158

55 Wrighton LJ, O’Bosky KR, Namm JP, Senthil M. Postoperative management after hepatic resection. J Gastrointest Oncol 2012; $3(1): 41-47$

56 Lisman T, Bakhtiari K, Pereboom IT, Hendriks HG, Meijers JC, Porte RJ. Normal to increased thrombin generation in patients undergoing liver transplantation despite prolonged conventional coagulation tests. J Hepatol 2010;52(3):355-361

57 Geerts WH, Pineo GF, Heit JA, et al. Prevention of venous thromboembolism: the Seventh ACCP Conference on Antithrombotic and Thrombolytic Therapy. Chest 2004;126(3, Suppl):338S-400S

58 Golse N, Bucur PO, Adam R, Castaing D, Sa Cunha A, Vibert E. New paradigms in post-hepatectomy liver failure. J Gastrointest Surg 2013;17(3):593-605

59 Friedman LS. Surgery in the patient with liver disease. Trans Am Clin Climatol Assoc 2010;121:192-204, discussion 205

60 Malinchoc M, Kamath PS, Gordon FD, Peine CJ, Rank J, ter Borg PC. A model to predict poor survival in patients undergoing transjugular intrahepatic portosystemic shunts. Hepatology 2000;31 (4):864-871

61 Northup PG, Wanamaker RC, Lee VD, Adams RB, Berg CL. Model for End-Stage Liver Disease (MELD) predicts nontransplant surgical mortality in patients with cirrhosis. Ann Surg 2005;242(2): 244-251

62 Farnsworth N, Fagan SP, Berger DH, Awad SS. Child-Turcotte-Pugh versus MELD score as a predictor of outcome after elective and emergent surgery in cirrhotic patients. Am J Surg 2004;188 (5):580-583

63 Perkins L, Jeffries M, Patel T. Utility of preoperative scores for predicting morbidity after cholecystectomy in patients with cirrhosis. Clin Gastroenterol Hepatol 2004;2(12):1123-1128

64 Befeler AS, Palmer DE, Hoffman M, Longo W, Solomon H, Di Bisceglie AM. The safety of intra-abdominal surgery in patients with cirrhosis: model for end-stage liver disease score is superior to Child-Turcotte-Pugh classification in predicting outcome. Arch Surg 2005;140(7):650-654, discussion 655 